\title{
Les consonances entre l'éthique jonassienne et la pensée confucéenne à l'âge écologique
}

Jean-Claude Gens

\section{(2) OpenEdition}

\section{Journals}

Édition électronique

URL : http://journals.openedition.org/alter/317

DOI : $10.4000 /$ alter.317

ISSN : 2558-7927

Éditeur :

Association ALTER, Archives Husserl (CNRS-UMR 8547)

\section{Édition imprimée}

Date de publication : 15 novembre 2014

Pagination : 247-262

ISBN : 978-2-9550449-0-2

ISSN : $1249-8947$

\section{Référence électronique}

Jean-Claude Gens, « Les consonances entre l'éthique jonassienne et la pensée confucéenne à l'âge écologique », Alter [En ligne], 22 | 2014, mis en ligne le 01 décembre 2017, consulté le 01 mai 2019. URL : http://journals.openedition.org/alter/317 ; DOI : 10.4000/alter.317

Ce document a été généré automatiquement le 1 mai 2019

Revue Alter 


\title{
Les consonances entre l'éthique jonassienne et la pensée confucéenne à l'âge écologique
}

\author{
Jean-Claude Gens
}

1 La corrélation entre l'éthique de la responsabilité de Jonas d'une part et les hypothèses théo-goniques ou -logiques de sa philosophie de la vie d'autre part, implique-t-elle une dépendance de la première vis-à-vis des secondes? C'est cette dépendance qui est interrogée dans le remarquable recueil co-édité en 2010 par Hava-Tirosh Samuelson et Christian Wiese The Legacy of Hans Jonas ${ }^{1}$. Si ce volume interroge surtout l'inspiration théologique juive de Jonas, William R. LaFleur a bien vu dans sa contribution que le paradigme de la responsabilité parentale consonne évidemment avec l'importance du rôle accordé par la pensée confucéenne à la relation père-fils. Cette consonance permet à ses yeux de comprendre la réception japonaise de sa pensée, et cette audience - qui contraste avec une réception limitée aux Etats-Unis - atteste, aux yeux de LaFleur, l'indépendance de la pensée de Jonas à l'égard d'une tradition théologique juive, c'est-àdire atteste la potentielle universalité de l'éthique jonassienne de la responsabilité. La thèse n'est par ailleurs pas sans intérêt au regard, plus généralement, de l'appel à dialoguer lancé par de nombreux penseurs confucéens aux philosophes occidentaux - un dialogue qui serait constitutif de ce que l'on convient parfois d'appeler la philosophie interculturelle. Il me semble que c'est aussi en ce sens que, considérant la détermination de la pensée par les traditions dont elle se nourrit, Jonas écrit : «Sans être lu, Descartes nous détermine, que nous le voulions ou non », pour ajouter :

Sans être lues, les Upanishads ne peuvent pas nous déterminer. Mais peut-être le devraient-elles? Alors nous devons les lire, précisément afin qu'elles puissent nous déterminer ${ }^{2}$.

Dans ce contexte, la thèse de LaFleur demande à être élargie à un double égard: il faut l'élargir au confucianisme en général et non seulement au confucianisme japonais, et l'élargir au cadre confucéen plus global dans lequel s'inscrit le thème de la relation parentale ou de la piété filiale. Il s'agira ici non pas de comparer, mais d'entendre des résonances, des consonances entre les pensées jonassienne et confucéenne qui se sont 
déployées séparément, ce qui permettra, par contraste, de mettre en relief la singularité de chacune de ces deux pensées. Sans suivre le fil chronologique de l'analyse de LaFleur, je me propose de 1) commencer par revenir sur le paradigme de la responsabilité parentale pour 2) considérer ensuite l'ambiguïté de son extension à la sphère politique et enfin 3) la possibilité d'entendre des consonances en ce qui concerne une dimension souvent secondarisée dans l'œuvre de Jonas, y compris par LaFleur: celle de notre responsabilité à l'égard de la nature.

3 Pour toute personne un tant soit peu familière avec la pensée confucéenne, il est impossible de rester insensible à sa consonance immédiate avec l'éthique jonassienne de la responsabilité en tant qu'elle a pour paradigme la responsabilité parentale. Que le rapprochement ne soit pas étrange, c'est ce que suggèrent également des contributions comme celle de Mary Evelyn Tucker à propos de la responsabilité intergénérationnelle. Comme le remarque Tucker, entendu à partir de l'éthique du Principe responsabilité le confucianisme est susceptible de s'élargir au-delà du sens de la continuité familiale et du sentiment de notre dette à l'égard des générations passées en direction du sentiment de notre dette envers les formes de vie qui nous ont précédé comme de notre responsabilité à l'égard des générations à venir et de notre environnement ${ }^{3}$. Le rapprochement a encore été tenté par William R. LaFleur dans une contribution intitulée «Infants, Paternalism, and Bioethics : Japan's Grasp of Jonas's Insistence on Intergenerational Responsibility ${ }^{4}$ parue en 2010 - une contribution à laquelle a répondu deux ans plus tard le professeur de philosophie et d'éthique de l'Université Kansai d'Osaka : Tetsuhiko Shinagawa.

4 LaFleur - dont les recherches ont en particulier porté sur l'esprit du Japon médiéval et qui a contribué à faire connaître l'École de Kyoto en Occident, mais qui a aussi publié sur la bioéthique au Japon - pointe l'asymétrie entre la réception japonaise et, plus largement, confucéenne et bouddhiste de Jonas et la sous-estimation de sa pensée aux États-Unis. LaFleur explique la réception japonaise par le fait que, « pour une bonne part, il n'y a là [dans l'éthique jonassienne] rien de si nouveau» pour les Japonais 5 . Considérant, comme Tucker, que le modèle de la responsabilité, qui permet de penser la responsabilité à l'égard des générations futures, ajoute une dimension temporelle à l'éthique kantienne, LaFleur estime que les pensées jonassienne et japonaise ont une « surprenante affinité ».

Une conception similaire à celle de Jonas se retrouverait ainsi au Japon bien avant la parution du Principe responsabilité, en particulier dans la pensée de Tetsurô Watsuji (1889-1960) qui est l'une des figures de la seconde génération de l'École de Kyoto. Insistant dans son Éthique (Rinrigaku) publiée en 1937 et révisée en 1962 sur la priorité du caractère relationnel de notre existence par rapport à la dimension personnelle et indépendante de notre subjectivité, Watsuji écrit ainsi :

Le fait de partir de la conscience du devoir-être d'un individu implique le risque de faire sombrer dans l'oubli des relations humaines qui sont néanmoins la base de cette conscience et de laisser l'éthique dégénérer en une science de la conscience subjective ${ }^{6}$.

Un oubli qui, pour Watsuji, caractérise aussi Être et temps de Heidegger dont il suivit les cours.

7 LaFleur pointe en particulier le fait que, pour Watsuji, ce qui est de manière factuelle est susceptible de signifier une obligation morale, un devoir-être, le philosophe japonais se référant alors précisément à l'enfant qui, sans témoigner encore d'une conscience individuelle, d'une volonté, etc., existe néanmoins immédiatement en relation à d'autres 
et en particulier à ses parents 7 . Watsuji défend dans ce contexte l'idée que l'éthique impliquée par ce que nous appelons le culte des ancêtres et par la piété filiale est orientée non seulement vers le passé, mais également vers le futur :

Dans notre conception de la famille [au Japon], ses membres ne se limitent pas à ceux qui sont actuellement vivants. Selon une ancienne coutume japonaise, chaque maison doit avoir un autel bouddhiste où l'on révère les ancêtres et, chaque année, à la date anniversaire d'un parent défunt, l'ensemble de la famille célèbre cet événement en observant des [pratiques] religieuses. À ce moment, même un parent en vie doit se comporter comme un enfant devant l'autel de parents défunts. Par conséquent on ne peut pas dire que le chef de la maisonnée le soit de manière absolue. De fait, il ne peut prendre de décisions arbitraires qui affecteraient sa famille, et cela car ses décisions sont guidées par les ancêtres et son devoir est de préserver la famille qui lui a été donnée par ses prédécesseurs. Son obligation, c'est de la transmettre à sa descendance sans lui avoir causé des torts ${ }^{8}$.

Une telle éthique « intergénérationnelle » est « inscrite dans le rite lui-même " ${ }^{9}$ alors que, comparativement, elle semble bien plus nouvelle et difficile à recevoir en Occident. Mais la proximité entre les pensées jonassienne et confucéenne est plus patente encore dans le cadre d'une corrélation souvent considérée comme problématique dans Le principe responsabilité: la corrélation entre la responsabilité parentale et la responsabilité de l'homme politique.

9 Comme on sait, après avoir commencé à expliciter la singularité de la responsabilité parentale ${ }^{10}$ en tant qu'elle est asymétrique, c'est-à-dire "verticale » (PR 135), Jonas introduit un second exemple de responsabilité, celui de l'homme politique (PR 136 sq.), mais sans pour autant les juxtaposer. Si ce sont des exemples bien différents, Le principe responsabilité n'en estime pas moins nécessaire de les considérer "ensemble ${ }^{11}$ pour dégager, à partir de leurs traits communs, un concept de responsabilité à la hauteur de notre époque, c'est-à-dire une responsabilité qui se caractérise non seulement par son unilatéralité et sa non-réciprocité, mais par sa totalité en tant qu'elle concerne toutes les dimensions de l'existence de l'enfant, par sa continuité en tant qu'elle ne peut pas «prendre de vacances » ou n'est pas résiliable, et par le fait qu'elle est relative à l'avenir en tant qu'elle vise à préserver les possibilités ${ }^{12}$.

Le privilège malgré tout accordé à la responsabilité parentale ${ }^{13}$ est attesté par la fin du chapitre spécifiquement consacré à la "théorie de la responsabilité », le chapitre quatre. Ce chapitre s'achève par un retour sur le caractère paradigmatique de l'enfant, et plus encore du nourrisson comme objet «originaire» de la responsabilité; plus qu'un exemple, la responsabilité parentale est «un exemple originaire » (Urbeispiel) qui est en réalité « l'archétype de toute responsabilité $~^{14} \mathrm{du}$ point de vue aussi bien de l'« évidence » que du « contenu » et en tant qu'il contiendrait ses différentes formes en "germe», pour s'étendre aujourd'hui «en direction d'autres horizons de responsabilité » $(\mathrm{PR}, 186)$ que commence par thématiser le chapitre suivant : les responsabilités vis-à-vis de l'humanité et de la nature. Or le caractère essentiellement vulnérable du vis-à-vis de la responsabilité est précisément aussi ce que disent certains passages des textes confucéens, y compris à propos de la responsabilité de l'homme politique.

11 L'idée que la sphère éthique du devoir n'est pas d'un autre ordre que celle de l'être, et que ce devoir procède de la perception d'un être vulnérable est patente dans le très célèbre passage du Mencius qui considère l'obligation signifiée par un enfant que nous verrions assis au bord d'un puits : 
Toute personne qui apercevrait aujourd'hui un petit enfant sur le point de tomber dans un puits, éprouverait en son cœur panique et douleur, non pas parce qu'il connaitrait ses parents, non pas pour acquérir une bonne réputation auprès des voisins ou amis, ni parce qu'il détesterait l'entendre pleurer. [...] Il serait inhumain de ne ressentir aucune commisération ${ }^{15}$.

Mais l'obligation impliquée par la perception du vulnérable est encore plus patente dans des passages où transparait la corrélation entre la relation père (et, ou mère)-fils, qui constitue la plus fondamentale des cinq grandes relations sociales : souverain-sujet, pèrefils, mari-femme, frère ainé-frère cadet, relations entre amis, d'une part, et le pouvoir politique d'autre part. Si le sinogramme qui signifie la piété filiale（xiào 孝） représente un enfant qui porte un vieillard sur son dos, Jonas retrouve au fond, du côté parental, une thèse, avancée par l'un des quatre Classiques confucéens : La grande étude (dàxué 大学). Ce classique déclare ainsi que «le prince doit commander à ses sujets avec la même bonté qu'un père a pour ses enfants »; mais l'importance, aux yeux de Jonas, de la vulnérabilité en tant qu'elle oblige est encore plus manifeste dans la suite de ce passage et dans le Mencius. La suite de ce passage, qui est une citation du Shüjing ou Classique des documents, précise la nature de la bonté dont doit faire preuve le prince : elle doit être du même ordre que « celle [de la mère] qui prend soin de son nourrisson $»^{16}$. Un passage du Mencius 3 A 5, et, en l'occurrence le mot de Maître Li, y revient dans des termes similaires : « Les Anciens gouvernaient comme s'ils tenaient un bébé dans les bras $»^{17}-$ une comparaison à mon sens sans équivalent dans la philosophie politique grecque lorsque celle-ci invoque le modèle de la relation paternelle.

Indépendamment du fait que la responsabilité de l'homme politique concerne bien moins l'avenir, le rapprochement opéré par Jonas entre les responsabilités paternelle et politique, leur analogie, est néanmoins non seulement problématique ${ }^{18}$, mais suspect au regard de la philosophie politique moderne. Sa problématicité tient à l'équivocité ou à l'évanescence de la relation parentale dans la mesure où la structure classique de la famille relève aujourd'hui plus d'une question que d'une évidence, y compris en Chine. Son caractère suspect tient au fait que le rapprochement entre les deux genres de responsabilité semble donner une nouvelle légitimité à un modèle de l'exercice du pouvoir politique qui, pour être classique, n'en n'a pas moins été rejeté par la philosophie politique occidentale moderne : le modèle du (bon) berger ou du pâtre, du père. Cette philosophie politique voit en effet dans ce modèle l'expression d'un paternalisme qui, indépendamment du danger de népotisme qu'il comporte, est exclusif de l'idéal démocratique occidental, bien que cet idéal soit en réalité en tension avec la réalité de l'exercice du pouvoir des "experts", c'est-à-dire bien que cet idéal soit souvent le paravent correct de la réalité technocratique de cet exercice.

Si LaFleur déplore également le caractère en général trop ambigu chez Jonas du rapprochement entre la responsabilité parentale et celle de l'homme politique ${ }^{19}$, on a d'autant plus pu suspecter la pensée de Jonas de défendre une conception paternaliste du pouvoir politique que, bien qu'il soit ensuite revenu de cette suggestion, la suite du Principe responsabilité suggère qu'une restriction des désirs illimités de consommation serait plus facile à penser dans le cadre d'États politiques autoritaires que dans celui des États démocratiques occidentaux, où l'ivresse de consommer répond aussi aux intérêts des entreprises de production. Jonas avance, comme on sait, qu'il faudrait qu'une « élite " intellectuelle et éthique puisse exercer une « tyrannie bienveillante » à l'égard des désirs - une tyrannie que seraient bien plus disposées à supporter les populations habituées aux régimes dits communistes $(\mathrm{PR}, 200)$. Jonas est revenu de cette hypothèse, par exemple 
dans son entretien de 1992 pour le Spiegel lorsque, en réponse au constat que dans les pays de l'ex bloc soviétique la nature a été dévastée, il avance :

Oui, c'est l'une des plus grandes déceptions. Je reconnais m'être entièrement trompé sur ce point. Je pensais que c'était du côté des communistes qu'il était le plus possible d'envisager une relation tempérante ou modérée à la nature, parce qu'ils pouvaient régir la satisfaction des besoins. Ils pouvaient dire ceci ou cela sera autorisé et pas plus ${ }^{20}$.

LaFleur rappelle que la dénonciation chez Jonas d'un paternalisme anti-démocratique par l'ouvrage de Richard Wolin, The children of Heidegger, a largement contribué à réduire la réception de la pensée de Jonas aux États-Unis ${ }^{21}$; mais la suspicion relative à l'extension du paradigme de la responsabilité paternelle à la sphère politique touche évidemment aussi l'importance du rôle de ce paradigme dans la pensée confucéenne, et elle s'est souvent traduite par la question de savoir si cette pensée est compatible avec les traditions démocratiques occidentales.

16 Cette question implique néanmoins de s'entendre sur ce que signifie la relation père-fils et la piété filiale, car les réserves occidentales tiennent en partie à l'ignorance de ce que cette relation signifie pour la pensée confucéenne ${ }^{22}$; l'individualisme de l'Occidental le conduisant à avoir une conception, pour ainsi dire, adolescente de cette relation, les parents apparaissent avant tout comme ceux dont il faut s'émanciper. Dans la pensée confucéenne en revanche, si le fils mu par un amour filial a - comme on l'a vu - pour tâche de continuer, de transmettre la volonté et l'œuvre du père, ce n'est pas sans avoir à interroger cette volonté explicite comme ses pensées secrètes et leur limitation ${ }^{23}$, ce qui comme le précise Tu Wei-ming, implique la possibilité d'une critique, certes respectueuse, des parents au regard de ce que demande le Ciel ${ }^{24}$. Plus encore, certains textes confucéens considèrent qu'à son plus haut degré la piété filiale consiste, pour chacun, à accomplir ce qui fait l'humanité de l'homme.

17 Neville rappelle en ce sens que l'amour parental constitue le champ par excellence où s'apprend et se teste l'amour, où il faut progressivement apprendre à accorder son autonomie à l'enfant, la lui apprendre, de telle sorte qu'il puisse lui aussi être parent un jour ${ }^{25}$.

18 Sur le plan politique, il convient de se souvenir que ce modèle ne justifie pas une forme de « despotisme oriental », puisque la révolte populaire témoigne, pour un confucéen, du fait que l'empereur s'est vu retirer le Mandat céleste au nom duquel il gouverne - ce qui constitue comme la version chinoise du fameux adage médiéval vox populi, vox dei. Par ailleurs, comme le rappelle $\mathrm{Tu}$ Wei-ming, l'extension du paradigme parental au politique implique d'aimer les membres de notre fratrie comme des enfants aimés de nos parents, de traiter nos voisins avec le respect qui honore l'amour que leur portent leurs parents, de traiter les concitoyens comme des enfants éloignés de l'empereur, et les barbares comme des personnes qui gagneraient beaucoup d'avoir un père comme notre empereur ${ }^{26}$ . Plus encore et conformément au Livre de la piété filiale qui précise que cette piété implique le respect $"$ de tous les pères dans le monde $»^{27}$, Wang Yang-ming considère que la véritable piété filiale suppose de ne plus faire qu'un corps avec les pères de tous les hommes :

C'est seulement lorsque j'aime mon père, les pères des autres, les pères de tous les hommes que mon humanité effective forme un seul corps avec mon père, les pères des autres et les pères de tous les hommes ${ }^{28}$. 
Dans « Why and How Has Hans Jonas Been "Welcomed" in Japan? A Reply from Japan to LaFleur's Interpretation» ${ }^{29}$, Tetsuhiko Shinagawa rend hommage à LaFleur en considérant qu'il y a un certain nombre de dimensions de la pensée de Jonas qui restent étrangères à celle des Japonais ${ }^{30}$, et qui empêchent donc de considérer, comme l'avance Lafleur, qu'il y ait une "affinité structurale » entre sa pensée et celle du Japon. Ainsi, l'orientation du culte des ancêtres vers l'avenir ne concerne, précise Shinagawa, qu'une famille donnée ${ }^{31}$ et non l'humanité. Bien qu'il ait factuellement raison au regard des pratiques confucéennes communes, cette restriction n'est pas inscrite dans la pensée confucéenne même comme le montre, par exemple, la manière dont, comme on vient de le voir, Wang Yang-ming invite à penser la piété filiale.

Inversement, le principe moral d'autonomie, tel qu'il a, par exemple, été thématisé par Kant, qui inspire la tradition politique de la démocratie souvent invoquée contre la pensée de Jonas n'en est pas moins lui-même problématique, car il marginalise, voire ignore, la spécificité de notre relation morale aux enfants, alors que ceux-ci constituent pourtant une partie importante de l'humanité. Est-il possible, demande LaFleur, de considérer les enfants comme une simple exception qui ne mériterait pas d'attention particulière dans le cadre d'une réflexion éthique? La chose est encore plus claire en ce qui concerne les enfants à naître qui ne sauraient donner ou refuser leur consentement à la manipulation de leur patrimoine génétique. LaFleur résume cette limite en citant MacIntyre :

Le point de départ de la plupart des philosophies morales présuppose toujours déjà l'existence de personnes indépendantes mâtures douées de la capacité à raisonner, et dont les relations sociales sont celles du monde adulte. L'enfance, à supposer qu'elle soit considérée, ne se voit accorder d'attention que de manière brève et incidente ${ }^{32}$.

21 En d'autres termes, en réduisant le cas des enfants et des nourrissons à de simples exceptions, la prétention de la morale classique à valoir universellement s'avère en réalité lacunaire. Les consonances entre la pensée confucéenne et celle de Jonas attestent aux yeux de LaFleur l'indépendance de cette dernière à l'égard de la, ou des, tradition(s) théologique(s) dans le cadre de laquelle elle s'est déployée. LaFleur ne se contente pourtant pas pour autant d'un tel raisonnement par l'absurde; il interroge encore la nature et les limites de la relation entre la pensée jonassienne et la pensée théologique juive. Mais pour considérer la potentielle universalité de la philosophie de Jonas, il faut élargir l'horizon de la réflexion de LaFleur qui est limité à la question de la responsabilité intergénérationnelle pour considérer les consonances entre cette philosophie et l'anthropocosmisme confucéen.

LaFleur part de la convergence entre les pensées de Jonas et de la tradition confucéenne pour mettre en question l'idée selon laquelle l'œuvre de Jonas se déploie à partir du fond de la tradition juive, c'est-à-dire en dépendrait ${ }^{33}$. On se souvient de la réappropriation par Jonas des doctrines du tsim-tsoum dans "L'immortalité et la mentalité moderne » (1962) puis dans Le concept de Dieu après Auschwitz et du reshimu, c'est-à-dire la doctrine selon laquelle des impressions résiduelles de la lumière divine perdurent dans l'espace cosmique $^{34}$ après que Dieu s'en soit retiré ${ }^{35}$. Même si Jonas a pu être inspiré par ces doctrines dont il avait connaissance au moins à travers Les grands courants de la mystique juive dont - comme l'a indiqué Christian Wiese - Gershom Scholem lui avait envoyé un exemplaire en $1942^{36}$, cela n'implique pas pour autant une "dépendance» de sa philosophie de la vie à leur égard, c'est-à-dire une limitation de la portée ou la validité de 
l'éthique jonassienne ${ }^{37}$. Car, en dehors du fait qu'une telle dépendance permettrait mal de comprendre la rapidité et l'ampleur de la réception japonaise de la pensée de Jonas, il n'est pas besoin de recourir à la religion pour constater, avance LaFleur, que

les hommes actuels sont en grande partie constitués par ce que furent jadis leurs ancêtres et que notre progéniture devra en grande partie ce qu'elle sera à ce qu'elle héritera de notre présent et de ce que nous sommes ${ }^{38}$.

Mettre en évidence le caractère contingent du lien entre l'éthique de la responsabilité de Jonas et les mythes théo-cosmogoniques, c'est-à-dire l'indépendance de la première par rapport à la seconde, est par ailleurs d'autant plus essentiel que la figure la plus en vue qui se réclame de Jonas aux États-Unis, Leon R. Kass, défend simultanément une éthique qui, elle, recourt à des arguments théologiques pour défendre des thèses bioéthiques relatives aux recherches sur les cellules souches et pour demander l'interdiction de l'avortement ${ }^{39}$. La distance ici de Jonas vis-à-vis de ce genre de position est manifeste, par exemple, dans un passage - cité par LaFleur - d'un entretien de Jonas avec le Spiegel du 11 mai 1992 intitulé « Dem bösen Ende näher » et dans lequel Jonas déclare que la position du pape Jean-Paul II contre le contrôle des naissances est «un crime contre la responsabilité à l'égard du monde $\aleph^{40}$. Cette distance est également claire à l'égard des nouvelles formes d'eugénisme que permet la biologie actuelle; la tentation de prendre des décisions dont l'impact concernera surtout des personnes, voire des populations, à venir, et de sélectionner sa progéniture « librement » ou de manière autonome grâce aux techniques nouvelles sont susceptibles de conduire à un paternalisme plus diffus et inavoué, mais non moins effectif, un paternalisme que Hisatake Katô - sous l'auspice duquel Le principe responsabilité a été traduit en japonais en 2000 - appelle « institué $»^{41}$.

Mais il convient maintenant d'élargir la perspective de LaFleur pour interroger les résonances entre l'arrière-plan mythique de l'éthique jonassienne et le contexte plus global de la pensée confucéenne, car la question de la responsabilité intergénérationnelle ne concerne que l'un des deux aspects de la pensée de Jonas, celui qui se soucie de l'humanité future. Qu'en est-il de la nature dont Jonas avance qu'elle a une valeur intrinsèque et ne demande pas à être considérée comme le simple moyen de conserver l'humanité, c'est-à-dire comme l'objet d'un devoir simplement indirect (PR, 187 sq.) ?

De manière générale, alors que la science moderne occidentale est physicomathématique, la compréhension chinoise du monde est plus inspirée par les phénomènes biologiques, les processus organiques, c'est-à-dire relève d'une « philosophie de l'organique $\gg^{42}$ pour reprendre l'expression de Joseph Needham ${ }^{43}$. À cet égard, la singularité de la pensée jonassienne de la vie par rapport à la pensée chinoise est double.

Ce qu'elle a de "nouveau », c'est d'abord qu'elle pense la vie en termes de subjectivité, d'une intérioritét ${ }^{44}$, bien que celle-ci puisse n'être que latente. La seconde spécificité de cette vie, c'est qu'elle est considérée dans le cadre d'une évolution au sens globalement darwinien de ce terme. Néanmoins l'idée d'une subjectivité latente dans les formes de vie les plus primitives, l'idée qu'il faut la penser en tant que tendance y compris dans le monde physique $(\mathrm{PR}, 109)$ ne saurait à mon sens être difficile à entendre pour un confucéen dans la mesure où il ne connaît pas le dualisme de l'esprit et de la matière. Par ailleurs, s'il n'y a pas de pensée confucéenne «évolutionniste ", il n'en reste pas moins que, comme on l'a souvent remarqué, la pensée chinoise est une pensée du devenir et non de l'être, ce qui signifie déjà de la transformation, de la mutation des choses. Et l'homme lui-même est conçu dans ce cadre comme le montre déjà le fait que le sinogramme désignant sa nature foncière 性 xìng inclut celui qui dit la vie, la génération et la 
croissance sheng 生 - shēng. Ce sinogramme, qui représente une plante qui croît, est aussi bien un nom désignant la vie elle-même qu'un verbe indiquant le procès de la génération, un terme qui dit la naissance et le fait de donner naissance. Si cette vie n'est pas sans pouvoir évoquer ce que les Grecs entendaient par phusis, elle est corrélée à une cosmologie bien différente ne serait-ce que par son souci de penser, là encore, une dynamique de la nature, dont témoigne par exemple la notion de qì, ou la conception des cinq éléments entendus non pas en tant qu'entités - à la manière des quatre éléments de la cosmologie grecque et de la médecine hippocratique -, mais comme autant de phases qui s'engendrent les unes les autres.

Cette vie, ces transformations, sont en même temps pensées en termes de créativité comme le montre, par exemple, le chapitre xxvi de L'invariable milieu ; ce chapitre insiste en effet sur le caractère « insondable » de la puissance « d'engendrement » du Ciel et de la Terre ; la suite de ce passage montre qu'il ne s'agit pas de penser cette puissance comme relevant d'une entité transcendante, et il oppose la façon dont nous percevons habituellement le ciel, la terre qui porte les dix mille êtres, la montagne, les eaux... d'une part, et leur puissance inaperçue d'engendrement d'autre part :

La voie suivie par le Ciel et la Terre est ample, consistante, haute, éclatante, se déploie au loin, s'étend en durée. De fait, le Ciel [n']est [que] cette quantité de lumière qu'on voit scintiller [...], mais, dans sa dimension infinie, le soleil, la lune et les astres y sont attachés et tous les existants en sont couverts ;

[...] de fait, la montagne n'est que cette quantité de pierre de la taille d'un poing, mais, dans son ampleur et sa vastité, les plantes et les arbres y poussent, oiseaux et quadrupèdes y ont leur séjour, et des trésors enfouis en sont extraits ${ }^{45}$.

L'essentiel tient ici à l'idée d'une "continuité » ${ }^{46}$ entre l'ordre cosmique et moral, politique, l'empereur n'étant lui-même que le Fils du Ciel - une continuité qui, comme chez Jonas, empêche de penser une rupture entre l'ordre des faits et celui des valeurs. Cette continuité, c'est-à-dire cette inscription de l'homme dans le cadre d'un cosmos, caractérise l'anthropocosmisme confucéen - pour reprendre cette expression que $\mathrm{Tu}$ Weiming emprunte lui-même à Mircea Eliade -, mais aussi jonassien. Et je repense ici à la critique par Jonas de «l'existentialisme » en tant qu'il signifie « un certain dualisme, une désunion entre l'homme et le monde, avec la perte d'un cosmos apparenté - bref, un acosmisme anthropologique $»^{47}$. Dans un contexte confucéen qui est évidemment étranger à l'avènement de la technique moderne, cet anthropocosmisme signifie néanmoins plus qu'une solidarité.

D'abord car la nature foncière de l'homme, ou du moins du sage qui s'est accordé au Ciel et à la Terre, participe également de la "pure créativité » cosmique, comme le rappelle Mou Zongsan ${ }^{48}$, du «ça travaille» de la nature comme dit Jonas (PR, 109), ou du travail d'enfantement de la création pour reprendre l'expression de l'Épitre aux Romains VIII, 8. Plus encore - et l'idée paraît évidemment « extravagante » comme le dit James Legge - la créativité des dix mille êtres procède aussi en retour de la créativité du saint ou du sage comme le suggère la suite de L'invariable milieu, c'est-à-dire son paragraphe XXVII : «La voie suivie par l'homme saint, comme elle est foisonnante! Elle répand et fait croître les dix mille êtres", ou encore: "Qu'elle est ample, la voie du sage! [...] elle déploie et nourrit tous les existants $»^{49}$. L'homme est ainsi appelé à collaborer, à assister le Ciel et la Terre de telle sorte qu'ils forment tous trois une «triade $»^{50}$. Un tel anthropocosmisme confucéen pourrait aussi entendre ou recevoir une idée dont il ne me souvient pas que Jonas la reprenne à son compte et qui ne renforcerait pas son éthique : l'idée selon laquelle l'homme aurait pour mandat ${ }^{51}$ de préserver et de sauver les étincelles de lumière 
qui subsistent dans l'espace libéré par la contraction divine du tsim-tsoum, le mandat décrit par Martin Buber comme étant constitutif de la kavana ${ }^{52}$.

Ce rapprochement entre les pensées jonassienne et confucéenne, qui vise à dessiner un cadre possible de dialogue entre la philosophie occidentale et la « littérature pensante » de l'Orient comme disait Merleau-Ponty, prend évidemment le risque de rendre l'œuvre de Jonas plus difficile à entendre pour certains de ses lecteurs - je pense, par exemple, à des lecteurs comme Jean-Pierre Changeux qui, dans son dialogue avec Ricœur, voyait déjà dans le recours au concept de fin en biologie « un dramatique retour » «à ce qu'il y a de plus contestable chez Aristote ${ }^{53}$. Quoi qu'il en soit de cette difficulté, à moins de récuser par avance la possible consistance du confucianisme, Jonas était manifestement loin de considérer la ou les traditions dont il s'était nourri avec suffisance, et il estimait au contraire qu' « aucune voix signifiante dans la symphonie humaine ne devrait manquer ", et cela d'autant plus qu' "il se pourrait que notre héritage occidental ne soit pas complètement exempt du besoin d'être corrigé ou complété $\aleph^{54}$. Il me semble que la pensée de Jonas donne au confucianisme la possibilité de penser la relation de l'homme à la nature aujourd'hui de manière plus décisive, dans la mesure où sa responsabilité doit pouvoir déterminer de manière nouvelle le sens de l'action politique. D'un autre côté, il n'est pas évident que l'éthique jonassienne de la responsabilité puisse se substituer aux éthiques traditionnelles. Cette éthique est en effet destinée à faire face, à répondre, à une menace, mais sans chercher - comme l'ont fait les sagesses antiques - à penser les conditions de l'avènement ou de l'accomplissement de l'humain dans les hommes, les conditions qui permettraient, pour ainsi dire, de les sauver d'eux-mêmes. De ce point de vue, l'éthique jonassienne resterait enfermée dans la sphère délimitée par Kant : celle du devoir, ou encore celle qui cherche une réponse à la question que dois-je faire ? La morale confucéenne cherche en revanche à penser une " authenticité ", une " sincérité » ou une sagesse qui consonne avec l'invitation, au premier abord étrange, de Jonas à éveiller ce qu'il appelle une "fidélité » (Treue) (PR, 188) ou une "piété » (Andacht) $)^{55}$ à l'égard de «l'ensemble des créations » $(\mathrm{PR}, 188)$ de la nature.

\section{NOTES}

1. H. Tirosh-Samuelson et Ch. Wiese éd., The Legacy of Hans Jonas. Judaism and Phenomenon of Life, Brill, Leiden/Boston, 2010.

2. Jonas, "Wandel und Bestand. Vom Grunde der Verstehbarkeit des Geschichtlichen ", in Philosophische Untersuchungen und metaphyische Vermutungen, Insel, 1992, p. 78, trad., "Changement et permanence. Sur la possibilité de comprendre l'histoire», in Essais philosophiques. Du credo ancien à l'homme technologique (1974), D. Bazin et O. Depré éd., Paris, Vrin, 2013, p. 331.

3. Mary Evelyn Tucker, "Confucianism and Ecology. Potential and Limits", in Earth Ethics 10, $n^{\circ} 1$ (Fall 1998), et plus largement M. E. Tucker, John Bertrong, éd., Confucianism and Ecology, Cambridge, Harvard University Press, 1998. 
4. William R. LaFleur, « Infants, Paternalism, and Bioethics: Japan's Grasp of Jonas's Insistence on Intergenerational Responsibility ", in The Legacy of Hans Jonas. Judaism and Phenomenon of Life, H. Tirosh-Samuelson et Ch. Wiese éd., Brill, Leiden/Boston, 2010, pp. 461-480.

5. LaFleur, op. cit., p. 462.

6. Watsuji Tetsurô's Rinrigaku: Ethics in Japan, Albany, State University of New York Press, 1996, p. 33, cité par LaFleur, op. cit., p. 471.

7. LaFleur, op. cit., p. 471.

8. Cité et traduit du japonais par LaFleur, op. cit., p. 472 ; on trouvera une traduction par B. Bernier et M. Richard de la section de Rinrigaku consacrée à l'État, in Laval Théologique et philosophique, 64, 2, juin 2008, pp. 345-357. En ce qui concerne cette conception de la piété filiale, voir L'invariable milieu, XIX, trad. R. Mathieu, La pratique équilibrée, in Philosophes confucianistes, Paris, Gallimard, « La Pléiade », 2009, pp. 585 sq.

9. LaFleur, op. cit., p. 473.

10. Jonas, Das Prinzip Verantwortung, Francfort-sur-le-Main, Suhrkamp, 1984, trad. J. Greisch, Le principe responsabilité - sauf exception, dorénavant cité PR dans le corps du texte -, Paris, Cerf, 1990, pp. 64 sq.; nous nous abstenons d'indiquer la pagination de l'édition allemande dans la mesure où elle figure dans le corps du texte de cette traduction.

11. "Il est du plus haut intérêt théorique de voir comment cette responsabilité issue du choix le plus libre et celle issue du rapport naturel le moins libre, celle de l'homme d'État et celle des parents [...] ont précisément le plus de choses en commun et que prises ensemble, elles peuvent nous en apprendre le plus long sur l'essence de la responsabilité », PR 139, souligné par Jonas.

12. Jonas, PR, 134, 145 et 150 sq.

13. Les Essais philosophiques vont jusqu'à voir dans cette responsabilité un présupposé de l'économie, op. cit, p. 134.

14. PR, 184 (je souligne, J.-C. Gens).

15. Mencius, 2 A, 6, trad. Lévy, Paris, Rivages, 2008, p. 85.

16. La grande étude 17, trad. Couvreur, in Textes essentiels de la pensée chinoise. Confucius et le confucianisme, A. Lavis éd., Presses Pocket, « Agora », 2008, p. 171, ou trad. R Mathieu, La pratique équilibrée, op.cit., p. 559. Comme le Mencius 3 A 3, où le prince est dit devoir être «le père et la mère du peuple ", ce passage montre que la relation dite du père au fils n'implique pas pour autant la négation du rôle de la mère.

17. Le livre de Mencius, 3 A 5, trad. A. Lévy, op. cit., p. 121, ou trad. Charles Le Blanc, in Philosophes confucianistes, op.cit., p. 369 : "Les anciens souverains gouvernaient le peuple "comme s'ils protégeaient un nouveau-né" ", ce qui est une citation du Shujing ou Livre des documents, XIV, 204 A.

18. Sur la critique de Arendt relative à la spécificité de la sphère politique, voir Jonas, Souvenirs, trad. S. Cornille, Ph. Ivernel, Paris, Payot-Rivages, 2005, p. 245.

19. LaFleur adoptant ici le point de vue non pas de R. Wolin qui critique ce qu'il pense être une simple réduction de la seconde à la première, mais de Richard Bernstein (LaFleur, op.cit., pp. 466-468).

20. « Dem bösen Ende näher », Der Spiegel, 1992, p. 107.

21. Richard Wolin, Heidegger's Children: Hannah Arendt, Karl Löwith, Hans Jonas, and Herbert Marcuse, Princeton University Press, 2001, p. 123.

22. Voir Lunyu II, 5 , où Confucius donne à ce propos des réponses divergentes à ceux qui le questionnent - le Xiaojing, le Livre de la piété filiale posant que cette piété est la racine de toutes les autres vertus et « la source » de l'enseignement (Philosophes confucianistes, op. cit., p. 618).

23. Tu Wei-Ming, Centrality and Commonality, Albany, State University of New York Press, 1989, p. 42, et voir 45, 53 bas et $105 \mathrm{sqq}$.

24. Centrality and Commonality, op. cit., p. 106 ; sur la nécessité de faire des remontrances à ses parents « avec réserve », voir Entretiens IV, 18, et le Livre de la piété filiale, qui applique à la relation 
entre le souverain et ses conseillers et ministres, le devoir de remontrance des fils (Philosophes confucianistes, op. cit., p. 641.

25. «Perhaps it is mainly in parenting that the profoundest lessons of boundary-setting and individuation are learned. Children, of course, get into trouble and dissapoint us. They get sick and sometimes die. In all this parental love is tested and grown», R. C. Neville, Boston Confucianism, Albany, State University of New York Press, 2000, p. 99.

26. Neville, op. cit., pp. 100 sq.

27. Philosophes confucianistes, op. cit., p. 639.

28. Wing-Tsit Chan, A Source book in Chinese Philosophy (1969), Princeton University Press, 1973, p. 660.

29. Tetsuhiko Shinagawa, "Why and How Has Hans Jonas Been "Welcomed" in Japan? A Reply from Japan to LaFleur's Interpretation ", in Journal of Philosophy of Life, Osaka, vol. 2, n 1, mars 2012 : pp. 15-31, où sont récapitulées les traductions japonaises de Jonas, dont la première, de 1986, concerne l'ouvrage sur la gnose. Voir sinon ses questions à LaFleur en 2009. En 1999, Shinagawa publiait en japonais "Nature, Environment and Human Being (in Arche, Kyoto, vol.7, pp. 145-154) dont on trouve entre temps une version anglaise sur la page de son site internet.

30. Tetsuhiko Shinagawa, op. cit., p. 26.

31. Tetsuhiko Shinagawa, "Why and How Has Hans Jonas Been "Welcomed" in Japan? », op. cit., p. 26.

32. Voir LaFleur, op.cit., p. 464, et Alasdair MacIntyre, Dependent Rational Animals: Why Human Beings Need the Virtues, Chicago, Open Court, 1999, p. 81.

33. LaFleur, op. cit., p. 473.

34. Voir G. Scholem, Les grands courants de la mystique juive (Die jüdische Mystik in ihren Hauptströmungen, Zurich, 1957, trad. M.-M. Davy, Paris, Payot, 1960), par exemple, pp. 279, 281 sqq ., qui, à propos du tsim-tsoum remarque que cette doctrine fait contrepoids au panthéisme (p. 279) et pointe sa proximité avec les thèses gnostiques (p. 285). Sur la subjectivité étendue par-delà l'animal au monde physique, voir encore PR, 109.

35. Voir R. Margolin, "Hans Jonas and Secular Religiosity ", in The Legacy of Hans Jonas, op. cit., p. 241. Sur l'idée d'une "subjectivité [...] latente dans le cosmos», voir, entre autres, l'introduction au Phénomène de la vie, p. 15, où Jonas parle de «tendance », puis " Materie, Geist und Schöpfung ", in Philosophischen Untersuchungen und metaphysische Vermutungen, Francfort-surle-Main, Insel, 1992, p. 231, trad. S. Cornille et Ph. Ivernel, "Matière, esprit et création ", in Évolution et liberté, Paris, Rivages, 2005, pp. 225 sq.

36. Voir Ch. Wiese, «Zionism, the Holocaust, and Judaism in a Secular World: New Perspectives on Hans Jonas's Frieensship with Gershom Scholem and Hannah Arendt ", in The Legacy of Hans Jonas, p. 167.

37. LaFleur se range en ce sens du côté de Lawrence Vogel (op. cit., pp. 474 sq.), dont il cite « Hans Jonas's Exodus: From German Existentialism to Post-Holocaust Theology », in Hans Jonas, Mortality and Morality: A Search for the Good after Auschwitz, L. Vogel éd., Evanston, Northwestern University Press, 1996, pp. 1-40.

38. LaFleur, op. cit., p. 475.

39. LaFleur, op. cit., pp. 476 sq.

Sur Kass, voir Lawrence Vogel, « Natural-Law Judaism? The Genesis of Bioethics in Hans Jonas, Leo Strauss and Leon Kass », in The Legacy of Hans Jonas, op. cit., pp. 287-324.

40. LaFleur, op. cit., p. 477, citant « Dem bösen Ende näher », op. cit., pp. 92-107, ici 103.

41. Lafleur, op. cit., p. 478.

42. Joseph Needham, Science and Civilisation in China, Cambridge University Press, 1956, tome 2, p. 291. 
43. Robin R. Wang, Yinyang. The Way of Heaven and Earth in Chinese Thought and Culture, Cambridge University Press, 2012, p. 14 (par exemple yang est généré (shēng) par yīn et inversement, p. 62, et c'est yinnyáng qui engendre (shēng), c'est-à-dire fait émerger, exister et durer les choses, p. 224 ; c'est aussi par rapport à shēng que le corps (shēn) est pensé, p. 185).

44. Jonas, Le phénomène de la vie, op. cit., p. 93.

45. L'invariable milieu XXVI, trad. F. Jullien, Zhong Yong. La régulation à usage ordinaire, Paris, Éditions de l'Imprimerie Nationale, 1993, p. 115.

46. Ibid., p. 18, et A. Cheng, Histoire de la pensée chinoise, Paris, Gallimard, « Folio », 2002, p. 444.

47. Jonas, Le phénomène de la vie, op. cit., p. 222.

48. Mou Zongsan, Spécificités de la philosophie chinoise, Paris, Cerf, 2003, entre autres pp. 219 et 221.

49. Respectivement R. Mathieu, La pratique équilibrée, op. cit., p. 594, et Jullien, Zhong Yong. La régulation à usage ordinaire, op. cit., p. 121.

50. L'invariable milieu, chap. 22, trad. Mathieu, La pratique équilibrée, op. cit., p. 591.

51. Jonas, Le phénomène de la vie, p. 277.

52. Buber, La légende du Baal-Shem, trad. H. Hildenbrand, Paris, Éd. du Rocher, 1984, p. 29.

53. J.-P. Changeux, P. Ricœur, Ce qui nous fait penser. La nature et la règle, Paris, Odile Jacob, Poches, 2000, p. 215.

54. Jonas, "Wandel und Bestand. Vom Grunde der Verstehbarkeit des Geschichtlichen », p. 79, trad., "Changement et permanence. Sur la possibilité de comprendre l'histoire", in Essais philosophiques, op. cit. p. 332.

55. Jonas, «Zur ontologischen Grundlegung einer Zukunftethik» (1985), in Philosophischen Untersuchungen und metaphysische Vermutungen, op. cit., p. 146, trad. S. Cornille et Ph. Ivernel, « Sur le fondement ontologique d'une éthique du futur », in Pour une éthique du futur, Paris, Rivages, 1998, p. 116. 